\title{
Cluster-glass behavior induced by local moment doping in the itinerant ferromagnet $\mathrm{Sc}_{3.1} \mathrm{In}$
}

\author{
E. Svanidze and E. Morosan \\ Department of Physics and Astronomy, Rice University, Houston, Texas 77005, USA
}

(Received 3 July 2013; published 14 August 2013)

\begin{abstract}
In the presented work, $\mathrm{Sc}_{3.1} \mathrm{In}$, a weak itinerant ferromagnet with no magnetic constituents, is doped with $\mathrm{Er}^{3+}$ local moment ions, to form $\left(\mathrm{Sc}_{1-x} \mathrm{Er}_{x}\right)_{3.1} \mathrm{In}$. As $x$ increases, the Weiss-like temperature $\theta$ stays positive and nearly triples up to $x=0.10$. Moreover, Er doping of as little as $x=0.02$ induces a cluster-glass state, which persists up to $x=0.10$, as evidenced by dc and ac susceptibility measurements, and confirmed by the Vogel-Fulcher analysis.

DOI: 10.1103/PhysRevB.88.064412

PACS number(s): 75.50.Lk, 75.10.Lp, 75.50.Cc
\end{abstract}

\section{INTRODUCTION}

While it has been shown that, in some magnetic systems, the nature of the magnetic moment can be changed from local to itinerant via pressure ${ }^{1}$ or doping, ${ }^{2,3}$ distinguishing between the two types of moments within a given system has proven to be difficult. ${ }^{4}$ A possible solution is to use a purely itinerant electron ferromagnet compound in which all constituents are nonmagnetic, and then titrate in local moment-bearing ions. Currently, only two compounds, $\mathrm{ZrZn}_{2}$ (Ref. 5) and $\mathrm{Sc}_{3}$ In (Ref. 6), are known to exhibit itinerant ferromagnetism despite the fact that their components do not possess any magnetic moment. An addition of local moment to the itinerant matrix was attempted via $\mathrm{Gd}^{3+}$ substitution in $\mathrm{Zr}_{1-x} \mathrm{Gd}_{x} \mathrm{Zn}_{2}{ }^{7}$ Surprisingly, it appeared that the instability of the itinerant magnetism in $\mathrm{ZrZn}_{2}$ (Ref. 6) prevented the enhancement of the overall magnetization upon doping. ${ }^{7}$ Moreover, the Curie temperature $T_{C}$ and the Weiss-like temperature $\theta$ decreased with increasing $x$ in $\mathrm{Zr}_{1-x} \mathrm{Gd}_{x} \mathrm{Zn}_{2}$, resulting in the suppression of the ferromagnetic state at $x_{c}=0.025$. $^{7}$ Perhaps not as surprising, nonmagnetic element doping also resulted in the suppression of the Curie temperature $T_{C} \cdot{ }^{8}$ The similarity between the magnetic and nonmagnetic doping effects is quite striking but could possibly be explained by the instability of ferromagnetism in $\mathrm{ZrZn}_{2}$, as predicted by the band structure with a narrow peak at the Fermi surface. ${ }^{6,9}$ For $\mathrm{Sc}_{3} \mathrm{In}$, the peak in the density of states at the Fermi level was found to be broader than that of $\mathrm{ZrZn}_{2} .{ }^{10}$ Attempts to drastically alter the overall magnetization via pressure ${ }^{11,12}$ and magnetic field ${ }^{13}$ were unsuccessful. However, $\mathrm{Lu}^{3+}$ doping resulted in remarkable non-Fermi-liquid (NFL) behavior, and revealed the existence of a quantum critical point (QCP) at $x_{c} \approx 0.03$. $^{14}$

The addition of local moment ions in itinerant magnets should provide an insight into the interplay between the two types of magnetism: the itinerant moment is expected to have a weak variation with the composition, but the overall magnetic moment should increase with increasing amounts of local moment. However, the itinerant magnetic moment was suppressed to zero upon Gd doping of $\mathrm{ZrZn}_{2}{ }^{7}$ Therefore, it is crucial to elucidate how the local and itinerant moments interact. To this end, the effects of Er doping into $\mathrm{Sc}_{3.1}$ In are reported here. In order to minimize the chemical pressure effects caused by doping, $\mathrm{Er}^{3+}\left(\mathrm{r}\left[\mathrm{Er}^{3+}\right]=0.890 \AA\right)$ (Ref. 15) was chosen since this magnetic rare earth was closest in size to the host $\mathrm{Sc}$ ion $\left(\mathrm{r}\left[\mathrm{Sc}^{3+}\right]=0.745 \AA\right)($ Ref. 15$)$ that it substituted for. Er doping as small as $x=0.02$ in $\left(\mathrm{Sc}_{1-x} \mathrm{Er}_{x}\right)_{3.1}$ In induced a cluster-glass state. The corresponding freezing temperature $T_{f}$ increased with increasing $x$ up to $x \approx 0.10$, a composition which appears to correspond to the solubility limit of Er in $\mathrm{Sc}_{3.1}$ In.

It has been established that the mechanism of the clusterglass behavior relies heavily on the existence of frustration and disorder. ${ }^{16}$ Antiferromagnetic coupling ${ }^{17-19}$ lends itself more readily to geometric frustration than ferromagnetic coupling, explaining the limited number of metallic, ferromagnetically coupled cluster-glass systems. Several cases are known, where a cluster-glass state arises from a ferromagnetic ground state in metal oxides, ${ }^{20-23}$ however, the metallic examples are limited to Pd-Mn (Ref. 24) and Ce-Ni-Cu (Ref. 25) alloys, $\mathrm{U}_{2} \mathrm{IrSi}_{3},{ }^{26} \mathrm{U}_{2} \mathrm{CoSi}_{3},{ }^{27}$ and $\mathrm{PrRhSn}_{3} .{ }^{28}$ While the cluster-glass state in both $\mathrm{U}_{2} \mathrm{IrSi}_{3}$ and $\mathrm{U}_{2} \mathrm{CoSi}_{3}$ has been attributed to crystallographic disorder, ${ }^{26,27}$ dynamic fluctuations of crystalfield levels have been suggested as the underlying mechanism for the magnetic frustration in $\operatorname{PrRhSn}_{3},{ }^{28}$ based on the fact that neither site disorder nor geometric frustration is present in this compound. The current study shows that the addition of $\mathrm{Er}^{3+}$ local moments in the ferromagnet $\mathrm{Sc}_{3.1} \mathrm{In}$ induces a cluster-glass state in $\left(\operatorname{Sc}_{1-x} \operatorname{Er}_{x}\right)_{3.1} \operatorname{In}(0<x \leqslant 0.10)$, while the Weiss-like temperature $\theta$, a measure of the local-to-itinerant moment coupling, remains positive. The Er-induced site disorder, along with frustration in the bipyramidal Sc/Er-In chains [inset, Fig. 1(a)] is the underlying source of the glassy behavior.

\section{EXPERIMENTAL METHODS}

It has already been established ${ }^{6}$ that the hexagonal $\mathrm{Sc}_{3} \mathrm{In}$ (space group $P 6_{3} / m m c$ ) forms nonstoichiometrically around the atomic ratio $\mathrm{Sc}: \mathrm{In}=3: 1$. In a previous study, ${ }^{14}$ it was determined that the optimal composition, which yielded the highest Curie temperature $T_{C}$ and paramagnetic moment $\mu_{\mathrm{PM}}^{\text {expt }}$, was $\mathrm{Sc}: \mathrm{In}=3.1: 1$. Polycrystalline samples of $\left(\mathrm{Sc}_{1-x} \mathrm{Er}_{x}\right)_{3.1} \mathrm{In}$ $(0 \leqslant x \leqslant 0.10)$ were prepared by arc melting Sc (Cerac 99.99\%), Er (Cerac 99.99\%), and In (Alfa Aesar, 99.9995\%), with mass losses no more than $0.3 \%$. The arc-melted buttons were subsequently wrapped in Ta foil and annealed under inert atmosphere for over two weeks at temperatures between $700{ }^{\circ} \mathrm{C}$ and $800^{\circ} \mathrm{C}$.

The arc-melted samples are extremely hard, comparable to some high carbon steels, ${ }^{29}$ making them difficult to cut or grind, thus rendering powder $\mathrm{x}$-ray diffraction experiments 

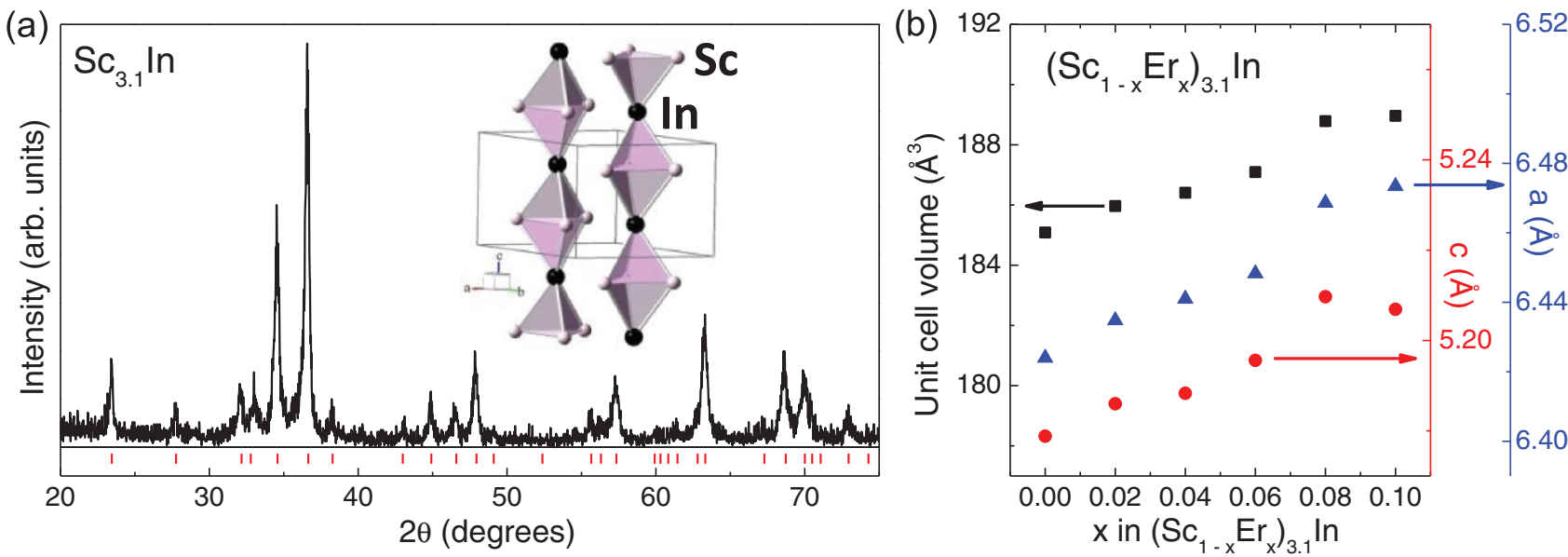

FIG. 1. (Color online) (a) X-ray diffraction pattern for $\mathrm{Sc}_{3.1} \mathrm{In}$, obtained from an arc-melted polycrystalline specimen (see text). Vertical marks correspond to the $P 6_{3} / m m c$ phase. Inset: nearly one-dimensional bipyramidal Sc-In chains. (b) Evolution of the lattice parameters $a$ (triangles), $c$ (circles), and the unit-cell volume $V$ (squares) with composition $x$.

very difficult. The surface of as-annealed samples was polished, and the $\mathrm{x}$-ray diffraction patterns were collected at room temperature off the cross section (about $5 \mathrm{~mm}$ in diameter) of these specimens, using a Rigaku D/Max diffractometer with $\mathrm{Cu} K \alpha$ radiation.

Zero-field-cooled (ZFC) and field-cooled (FC) dc magnetization measurements for the annealed samples were performed in a Quantum Design (QD) Magnetic Property Measurement System (MPMS) for temperatures from 2 to $300 \mathrm{~K}$. The ac susceptibility measurements (for frequencies $f$ between 10 and $10^{4} \mathrm{~Hz}$ ) were carried out from 2 to $50 \mathrm{~K}$ in a QD Physical Property Measurement System (PPMS). Specificheat measurements above $0.4 \mathrm{~K}$ were also performed, using an adiabatic relaxation method, in the QD PPMS.

\section{RESULTS AND ANALYSIS}

The $P 6_{3} / m m c$ structure and purity of the $\left(\mathrm{Sc}_{1-x} \mathrm{Er}_{x}\right)_{3.1} \mathrm{In}$ samples for $0 \leqslant x \leqslant 0.10$ was confirmed by $\mathrm{x}$-ray diffraction measurements. The lattice parameters were determined using GSAS (General Structure Analysis System) refinement. In the case of undoped $\mathrm{Sc}_{3.1}$ In [Fig. 1(a)], the lattice parameters $a=6.42 \AA$ and $c=5.18 \AA$ agree with the previously reported values. ${ }^{30}$ The composition dependence of the lattice parameters $a$ (triangles) and $c$ (circles), together with the change in the unit-cell volume $V$ (squares), is shown in Fig. 1(b) for $\left(\mathrm{Sc}_{1-x} \mathrm{Er}_{x}\right)_{3.1} \operatorname{In}(0 \leqslant x \leqslant 0.10)$. A systematic increase in the lattice parameters $a$ and $c$, and the unit-cell volume $V$ is observed with increasing Er concentration. Secondary phase peaks become visible in the $\mathrm{x}$-ray data for $x>0.10$, suggesting that this is the solubility limit for Er in this hexagonal structure.

The dc magnetic susceptibility presented in Fig. 2(a) shows irreversibility between ZFC (full symbols) and FC (open symbols) data at low temperatures. This irreversibility, together with the increase of the FC magnetization upon cooling, is likely associated with either a cluster-glass state or a long-range ferromagnetic order. The ac susceptibility data presented below points to the cluster-glass scenario.
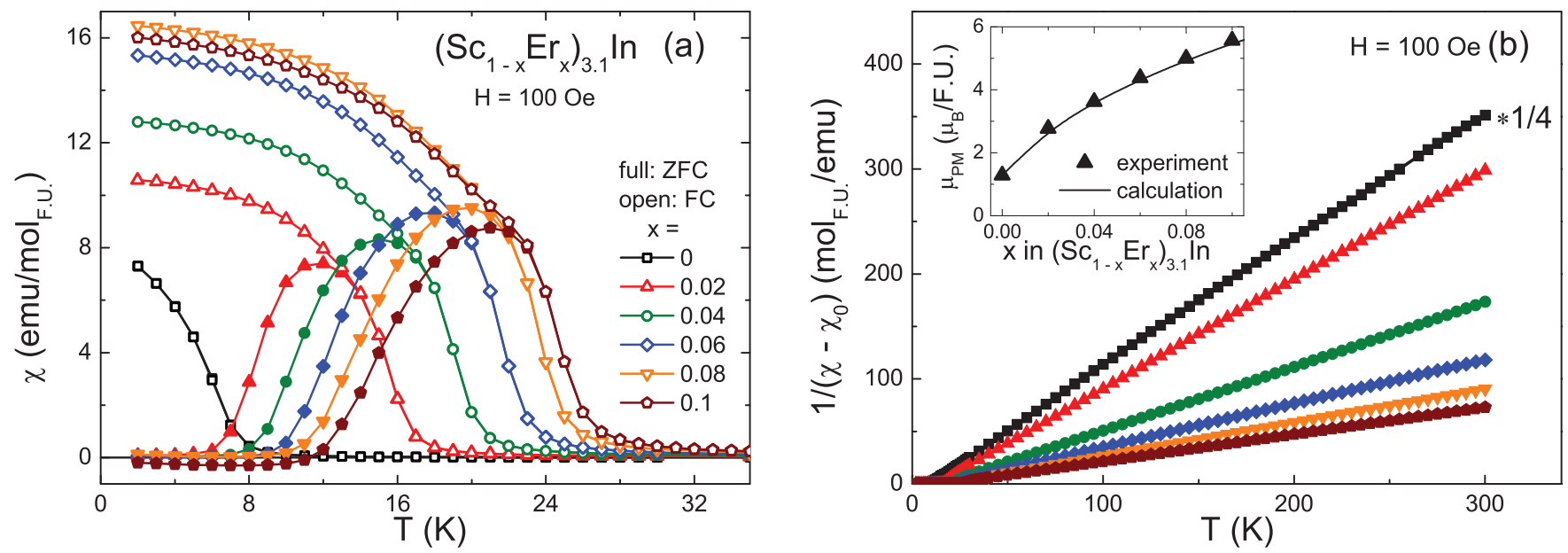

FIG. 2. (Color online) (a) ZFC (full) and FC (open) dc susceptibility for $\left(\operatorname{Sc}_{1-x} \operatorname{Er}_{x}\right)_{3.1} \operatorname{In}(0 \leqslant x \leqslant 0.10)$. (b) Inverse susceptibility for $\left(\mathrm{Sc}_{1-x} \mathrm{Er}_{x}\right)_{3.1}$ In where $0 \leqslant x \leqslant 0.10$. The $x=0$ data are scaled by a factor of $\frac{1}{4}$. Inset: composition dependence of measured (triangles) and calculated (line) paramagnetic moments $\mu_{\mathrm{PM}}^{\text {expt }}$ and $\mu_{\mathrm{PM}}^{\mathrm{calc}}$. 
At high temperatures, the temperature-dependent susceptibility should be analyzed in the context of the interplay between local and itinerant moment magnetism. For local moments, the susceptibility $\chi_{L}$ ( $T$ is described by the CurieWeiss law), ${ }^{31}$

$$
\chi_{L}(T)=\chi_{0, L}+\frac{C_{L}}{T-\theta_{W}},
$$

where $\chi_{0}$ is the temperature-independent magnetic susceptibility, $C_{L}$ is the local moment Curie constant, and $\theta_{W}$ is the Weiss temperature. The itinerant moment susceptibility $\chi_{I}(T)$ also varies inversely proportional to the temperature, ${ }^{31}$ and, in the case of strong spin fluctuations, $\chi_{I}(T)$ can be written as $^{14,31}$

$$
\chi_{I}(T)=\chi_{0, I}+\frac{C_{I}}{T-T_{C}^{*}} .
$$

Here, $C_{I}$ is a Curie-type constant, proportional to the quadratic paramagnetic itinerant moment, and $T_{C}^{*}$ is a Weisslike temperature. For a system with mixed local and itinerant moment contributions, their linear superposition would result in a magnetic susceptibility of the form

$$
\chi(T)=\chi_{0}+a \frac{C_{L}}{T-\theta_{W}}+b \frac{C_{I}}{T-T_{C}^{*}} .
$$

As seen in Fig. 2(b), the inverse high-temperature susceptibility $1 /\left[\chi(T)-\chi_{0}\right]$ is linear in $T$ for $x \leqslant 0.10$, which is consistent with Eq. (3) only if $\theta_{W}=T_{C}^{*}=\theta$, suggesting cooperative behavior of the local and itinerant moments in $\left(\mathrm{Sc}_{1-x} \mathrm{Er}_{x}\right)_{3.1} \mathrm{In}$ :

$$
\Delta \chi(T)=\chi(T)-\chi_{0}=\frac{x C_{L}+(1-x) C_{I}}{T-\theta}=\frac{C_{\mathrm{tot}}}{T-\theta} .
$$

The paramagnetic moment $\mu_{\mathrm{PM}}^{\mathrm{exp}}$ and the Weiss-like temperature $\theta$ can be determined from the linear fits of the inverse susceptibility at high temperatures. The $\theta$ values, listed in Table I, remain positive and increase monotonously with $x$ up to $x=0.10$, in contrast with the Gd-doped $\mathrm{ZrZn}_{2}$ where $\theta$ values decreased with Gd concentration. ${ }^{7}$ Despite the fact that the $x=0$ sample has no magnetic constituents, the value of its paramagnetic moment $\mu_{\mathrm{PM}}^{\mathrm{exp}}(\mathrm{x}=0)=\mu_{I} \approx 1.3 \mu_{B} /$ f.u. is remarkably large, consistent with previous reports. ${ }^{6,13,14}$ As the amount $x$ of Er is increased, the overall paramagnetic moment $\mu_{\mathrm{PM}}^{\text {expt }}$ grows, as indicated by the decreasing slope of the inverse susceptibility in Fig. 2(b). The calculated paramagnetic moment $\mu_{\mathrm{PM}}^{\text {calc }}$ can be estimated as a function of $x$ :

$$
\mu_{\mathrm{PM}}^{\mathrm{calc}}=\sqrt{(1-x) \mu_{I}^{2}+3.1 x \mu_{L}^{2}}
$$

where $\mu_{I}=1.3 \mu_{B} /$ f.u. is the itinerant contribution and $\mu_{L}=9.59 \mu_{B} / \mathrm{Er}^{3+}$ is the local moment per $\mathrm{Er}^{3+}$ ion. Good agreement between $\mu_{\mathrm{PM}}^{\text {expt }}$ and $\mu_{\mathrm{PM}}^{\text {calc }}$ is revealed in the inset of Fig. 2(b), an indication of cooperative behavior of the local and itinerant moments in this system.

In order to verify the glassiness in $\left(\mathrm{Sc}_{1-x} \mathrm{Er}_{x}\right)_{3.1} \mathrm{In}$, as suggested by the dc magnetization data above, additional ac and dc magnetization, and specific-heat measurements have been performed. All glassy systems are expected to exhibit (i) the bifurcation of the ZFC and FC dc magnetic susceptibility below the freezing temperature $T_{f}(0)$, (ii) a frequency-dependent maximum in the ac susceptibility $\chi^{\prime}(T)$, and (iii) a broad cusp in the temperature-dependent specific heat $C_{p}(T)$ at $T \approx 1.3 T_{f}(0)$. Cluster-glass materials are glassy systems in which the spins exhibit short-range correlations within a cluster, while the clusters themselves show the cooperative freezing characteristic of spin glasses. Such cluster-glass systems display (iv) an increase in the temperature-dependent FC susceptibility $\chi(T)$ below $T_{f}(0),(\mathrm{v})$ lack of saturation in $M(H)$ at high magnetic fields, and (vi) freezing temperatures, defined as maxima in the dc ZFC $\chi(T)$ data, generally lower than the irreversibility temperatures $T_{\text {irr }}$ between the ZFC and FC data. ${ }^{16,32}$ The dc magnetization measurements on $\left(\mathrm{Sc}_{1-x} \mathrm{Er}_{x}\right)_{3.1}$ In have already revealed some signatures of cluster-glass behavior, including (i) ZFC-FC irreversibility below $T_{\text {irr }}$, (iv) increasing $\chi_{\mathrm{FC}}$ on cooling, and (vi) $T_{f}(0)<T_{\text {irr }}$ [Fig. 2(b)]. The ac susceptibility $\chi^{\prime}(T)$ and specific-heat data, together with the magnetization isotherms $M(H)$ at $T=2 \mathrm{~K}$, presented below, reveal additional traits associated with the cluster-glass behavior in $\left(\mathrm{Sc}_{1-x} \mathrm{Er}_{x}\right)_{3.1} \mathrm{In}(0.02 \leqslant x \leqslant 0.10)$.

A notable effect of Er doping in $\mathrm{Sc}_{3.1}$ In is large hysteresis and finite coercivity in $\left(\mathrm{Sc}_{1-x} \mathrm{Er}_{x}\right)_{3.1}$ In $(0.02 \leqslant x \leqslant 0.10$, Fig. 3), while in the pure itinerant system ( $x=0$, squares, Fig. 3) no hysteresis is observed at $T=2 \mathrm{~K}$. It has been remarked $^{33}$ that the low-temperature magnetic moment in a cluster glass is often less than the one for the saturated single-ion moment. The field-dependent magnetization data, shown in Fig. 3, can be used to determine $\mu_{5.6} \mathrm{~T}$ as the lower limit for the saturated magnetic moment for all compositions,

\begin{tabular}{|c|c|c|c|c|c|c|c|}
\hline$x$ & $\begin{array}{l}T_{\text {irr }} \\
(\mathrm{K})\end{array}$ & $\begin{array}{c}\mu_{\mathrm{PM}}^{\operatorname{expt}} \\
\left(\mu_{B} / \text { f.u. }\right)\end{array}$ & $\begin{array}{c}\theta \\
(\mathrm{K})\end{array}$ & $\delta$ & $\begin{array}{c}T_{f}(0) \\
(\mathrm{K})\end{array}$ & $z v$ & $\begin{array}{c}T_{0} \\
(\mathrm{~K})\end{array}$ \\
\hline 0 & & $1.29 \pm 0.05$ & $6.4 \pm 1$ & & & & \\
\hline 0.02 & $18 \pm 1$ & $2.77 \pm 0.05$ & $13.0 \pm 1$ & 0.014 & $15.1 \pm 1$ & $2.7 \pm 2$ & 14.1 \\
\hline 0.04 & $19 \pm 1$ & $3.62 \pm 0.05$ & $16.5 \pm 1$ & 0.012 & $17.6 \pm 1$ & $11.0 \pm 2$ & 15.6 \\
\hline 0.06 & $22 \pm 1$ & $4.38 \pm 0.05$ & $15.9 \pm 1$ & 0.010 & $20.6 \pm 1$ & $8.8 \pm 2$ & 18.7 \\
\hline 0.08 & $24 \pm 1$ & $5.00 \pm 0.05$ & $17.4 \pm 1$ & 0.009 & $22.2 \pm 1$ & $10.1 \pm 2$ & 20.0 \\
\hline 0.10 & $25 \pm 1$ & $5.57 \pm 0.05$ & $17.2 \pm 1$ & 0.009 & $22.9 \pm 1$ & $10.8 \pm 2$ & 20.6 \\
\hline
\end{tabular}
which can then be compared to the calculated values. The calculated saturated moment is found as the superposition of the itinerant $\mu_{\text {sat }}^{\text {calc }}$ contribution from $\mathrm{Sc}_{3.1} \mathrm{In}$, and the local $\mathrm{Er}^{3+}$

TABLE I. Cluster-glass parameters for $\left(\mathrm{Sc}_{1-x} \operatorname{Er}_{x}\right)_{3.1} \operatorname{In}(0 \leqslant x \leqslant 0.10)$. 


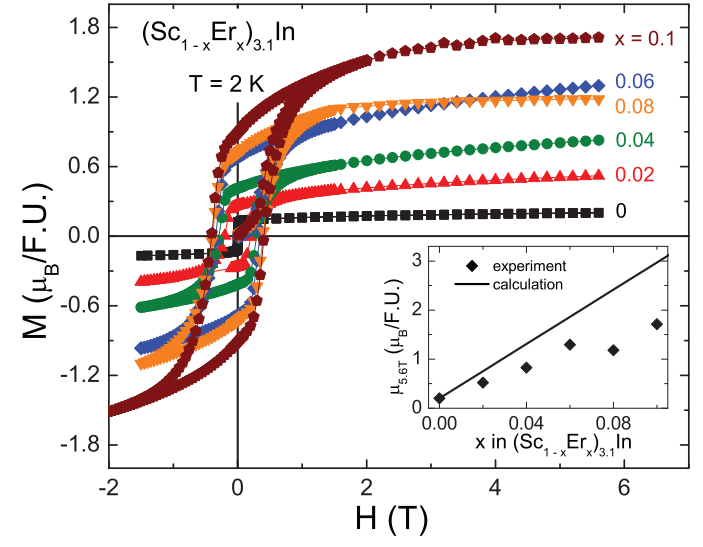

FIG. 3. (Color online) $T=2 \mathrm{~K}$ magnetization as a function of field for $\left(\operatorname{Sc}_{1-x} \operatorname{Er}_{x}\right)_{3.1}$ In with $0 \leqslant x \leqslant 0.10$. Inset: calculated saturated moment $\mu_{\mathrm{sat}}^{\text {calc }}$ (line) and measured magnetic moment $\mu_{5.6 \mathrm{~T}}$ (diamonds).

contribution, scaled per formula unit:

$$
\mu_{\text {sat }}^{\text {calc }}(x)=3.1 x \mu\left(\operatorname{Er}^{3+}\right)+(1-x) \mu\left(\operatorname{Sc}_{3.1} \mathrm{In}\right),
$$

where $\quad \mu\left(\mathrm{Er}^{3+}\right)=g J \mu_{B}=9 \mu_{B}, \quad$ and $\quad \mu\left(\mathrm{Sc}_{3.1} \mathrm{In}\right)=$ $0.20 \mu_{B}$ /f.u. is the magnetic moment of $\mathrm{Sc}_{3.1}$ In at maximum field available for these measurements $(H=5.6 \mathrm{~T})$. For example, for $x=0.10$, the largest measured magnetic moment, taken as the $M(H)$ value at $5.6 \mathrm{~T}$ (Fig. 3), is $\mu_{5.6 \mathrm{~T}}(x=0.10)=1.71 \mu_{B}$. For $x>0$, the calculated values of $\mu_{\text {sat }}^{\text {calc }}(x)$ are larger than the measured $\mu_{5.6 \mathrm{~T}}$ ones, as seen in the inset of Fig. 3, consistent with the cluster-glass state. ${ }^{33}$

For $0.02 \leqslant x \leqslant 0.10, \chi^{\prime}(T)$ (Fig. 4) reveals a broad, frequency-dependent peak, another indicator of glassy behavior. ${ }^{16}$ Conversely, no peak and no frequency dependence can be detected for $x=0$, indicating that doping is necessary to induce glassiness. Fits to the measured $\chi^{\prime}(T)$ peaks, with an example shown in Fig. 4(d) (solid line), yield values of the freezing temperature $T_{f}(f)$, which are plotted in Fig. 5 . A parameter $\delta$ can be determined from the change in the frequency $f$ with the freezing temperature $T_{f}(f) .{ }^{16}$ This is a quantitative measure of peak shift in $\chi^{\prime}(T)$ with frequency, and it is used to discriminate between spin-glass, cluster-glass, and superparamagnetic systems:

$$
\delta=\frac{\Delta T_{f}(f)}{T_{f}(f) \Delta(\ln f)} .
$$

For $0.02 \leqslant x \leqslant 0.10$ in $\left(\mathrm{Sc}_{1-x} \mathrm{Er}_{x}\right)_{3.1} \mathrm{In}$, the $\delta$ values are around 0.01 , higher than those reported for typical canonical spin glasses $\left(\delta \approx 0.005\right.$ for $\left.\mathrm{Cu}_{1-x} \mathrm{Mn}_{x}\right),{ }^{16,32}$ and lower than those for noninteracting ideal superparamagnetic systems $(\delta>0.10),{ }^{34}$ but comparable to those of known cluster-glass compounds $\left(\mathrm{PrRhSn}_{3} \text { and } \mathrm{CeNi}_{1-x} \mathrm{Cu}_{x}\right)^{25,28}$ This provides more evidence for a cluster-glass state induced by Er doping in $\mathrm{Sc}_{3.1} \mathrm{In} .{ }^{16}$

In a cluster glass, the relaxation time $\tau$ is a measure of the proximity to the spin-glass limit. ${ }^{32}$ The power law, established by Hohenberg et al., ${ }^{35}$ is frequently used to describe this behavior: ${ }^{16,32}$

$$
\tau=\tau_{0}\left(\frac{T_{f}(f)}{T_{f}(0)}-1\right)^{z v},
$$

where $\tau_{0}$ is the characteristic relaxation time of a single cluster, $v$ is the critical exponent which describes the growth of the correlation length $\xi\left\{\xi=\left[T_{f}(f) / T_{f}(0)-1\right]^{-\nu}\right\}$, and $z$ is the dynamic exponent which describes the evolution of the relaxation time $\left(\tau \propto \xi^{z}\right)$. The power-law fits, shown in Figs. 5(a)-5(e) for $\left(\mathrm{Sc}_{1-x} \mathrm{Er}_{x}\right)_{3.1}$ In for $0.02 \leqslant x \leqslant 0.10$, are used to determine the parameters $z v$ and $\tau_{0}$. Common values of the characteristic relaxation time $\tau_{0}$ in glassy systems are $\sim 10^{-12} \mathrm{~s}$, comparable to that obtained for $\left(\mathrm{Sc}_{1-x} \mathrm{Er}_{x}\right)_{3.1} \mathrm{In}$.
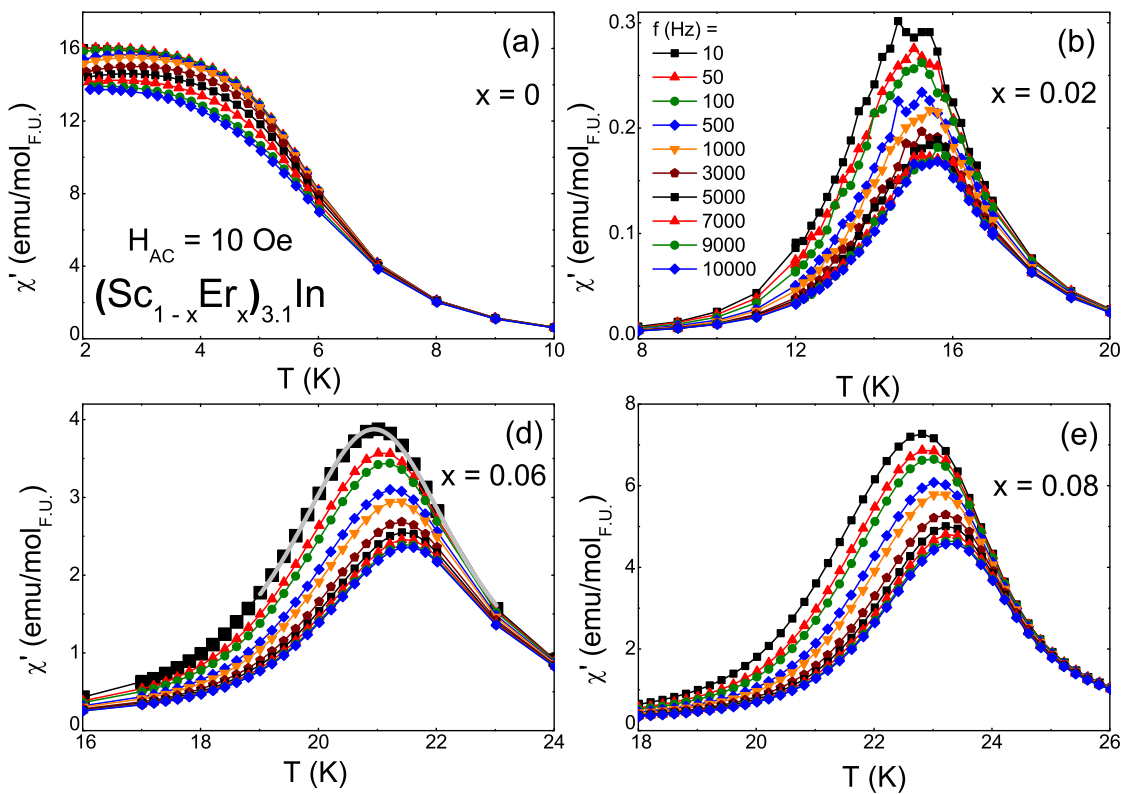
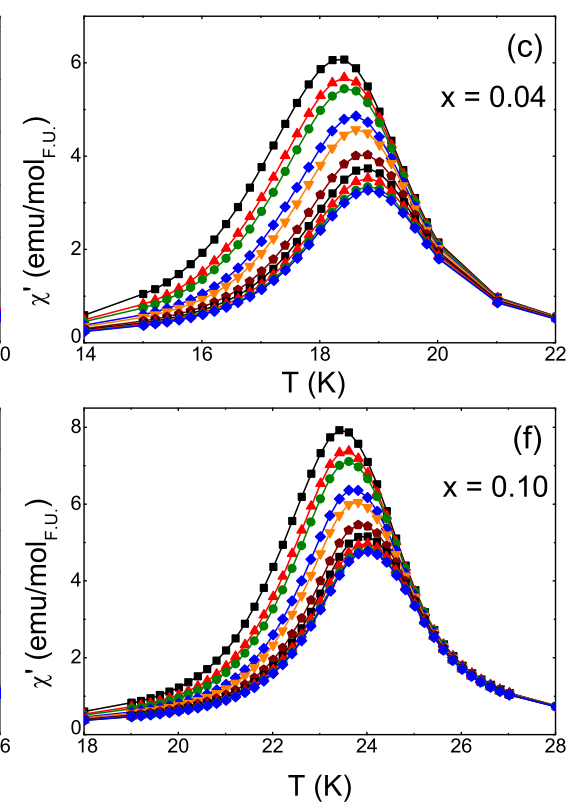

FIG. 4. (Color online) Frequency dependence of the real component $\chi^{\prime}(T)$ of the ac susceptibility data for $\left(\operatorname{Sc}_{1-x} \operatorname{Er}_{x}\right)_{3.1} \operatorname{In}$ with $0 \leqslant x \leqslant$ 0.10. An example of fit used to determine the freezing temperature $T_{f}(f)$ is shown in panel (d) for $f=10 \mathrm{~Hz}$. 

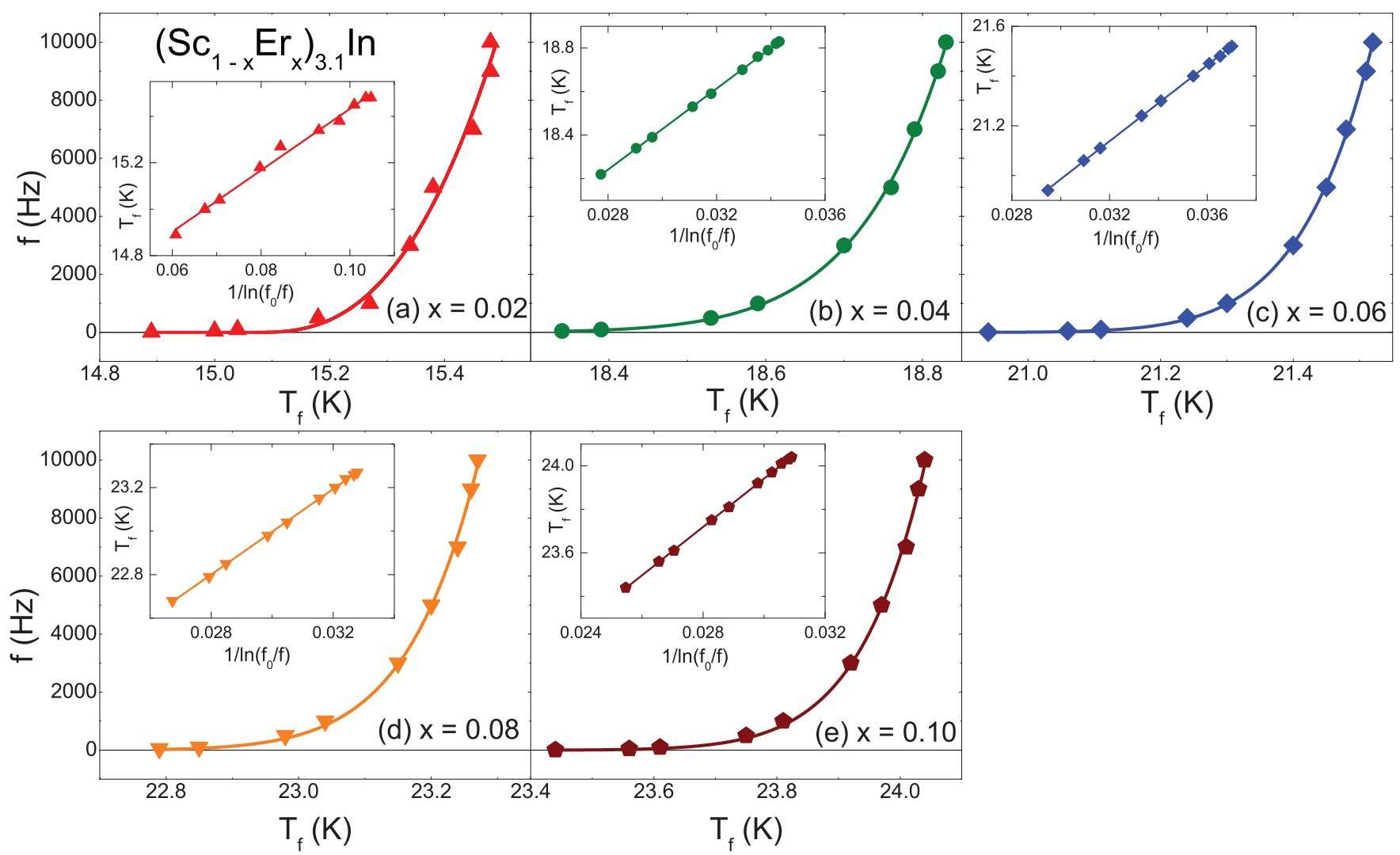

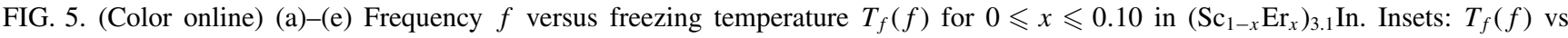
$1 / \ln \left(f_{0} / f\right)$ along with the fits (solid lines) to the Vogel-Fulcher law (see text).

Empirically, the $z v$ values for glassy systems have been observed to fall within the range $2 \leqslant z v \leqslant 14 .^{36}$ This is also true in the case of $\left(\mathrm{Sc}_{1-x} \mathrm{Er}_{x}\right)_{3.1} \mathrm{In}$, as the $z v$ values, listed in Table I, are close to 10 for most $x \leqslant 0.10$ samples, and smaller (but still $>2$ ) only for $x=0.02$.

Using the values of the relaxation time $\tau_{0}$ determined above, the characteristic frequency $f_{0}$ is calculated as $f_{0}=1 /\left(2 \pi \tau_{0}\right)$. The relationship between the freezing temperature $T_{f}(f)$ and the characteristic frequency $f_{0}$ is given by the empirical VogelFulcher law ${ }^{16,36-38}$ which takes into consideration the strength of intercluster interactions ${ }^{28,39}$

$$
f=f_{0} e^{-\frac{E_{a}}{k_{B}\left(T_{f}(f)-T_{0}\right)}} .
$$

Here $E_{a}$ is the activation energy, i.e., the energy that the clusters must overcome in order to align with the magnetic field $H, k_{B}$ is the Boltzmann constant, and $T_{0}$ is the VogelFulcher temperature that is often interpreted as a measure of intercluster interaction strength. ${ }^{16,27,28,40}$ It is possible to fit the data with the above equation rewritten as

$$
T_{f}(f)=\frac{E_{a}}{k_{B}} \frac{1}{\ln \left(f_{0} / f\right)}+T_{0} .
$$

The parameters $E_{a}$ and $T_{0}$, derived, respectively, from the slope and intercept of the Vogel-Fulcher fits of $T_{f}(f)$ versus $1 / \ln \left(f_{0} / f\right)$ [shown in the insets of Figs. 5(a)-5(e)], are summarized in Table I. The nonzero values of $T_{0}$ (Refs. 28 and 39) confirm that the clusters are strongly correlated, while
$T_{0} \leqslant 0$ would correspond to a collection of noninteracting spins, i.e., a spin-glass compound.

The specific-heat data for $\left(\mathrm{Sc}_{1-x} \mathrm{Er}_{x}\right)_{3.1}$ In $(0.02 \leqslant x \leqslant$ $0.10)$, plotted as $C_{p} / T$ versus $T^{2}$ in Fig. 6, displays a broad peak near the freezing temperature $T_{f}(0)$, marked by the large open symbols. A broad maximum at the temperature exceeding the freezing temperature $T_{f}(0)$ is usually observed in cluster-glass systems, as mentioned above. No such peak is visible for $x=0$, although at low $T$ the upturn in $C_{p} / T$ is thought to be associated with NFL behavior. ${ }^{14}$ Even for

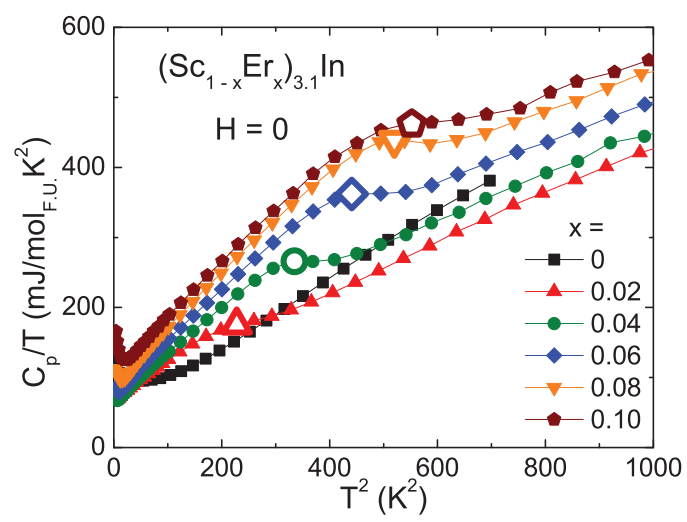

FIG. 6. (Color online) $C_{p} / T$ vs $T^{2}$ for $\left(\mathrm{Sc}_{1-x} \mathrm{Er}_{x}\right)_{3.1} \operatorname{In}(0 \leqslant x \leqslant$ $0.10)$, with open symbols corresponding to the freezing temperature $T_{f}(0)$, as determined from $\chi^{\prime}(T)$. 

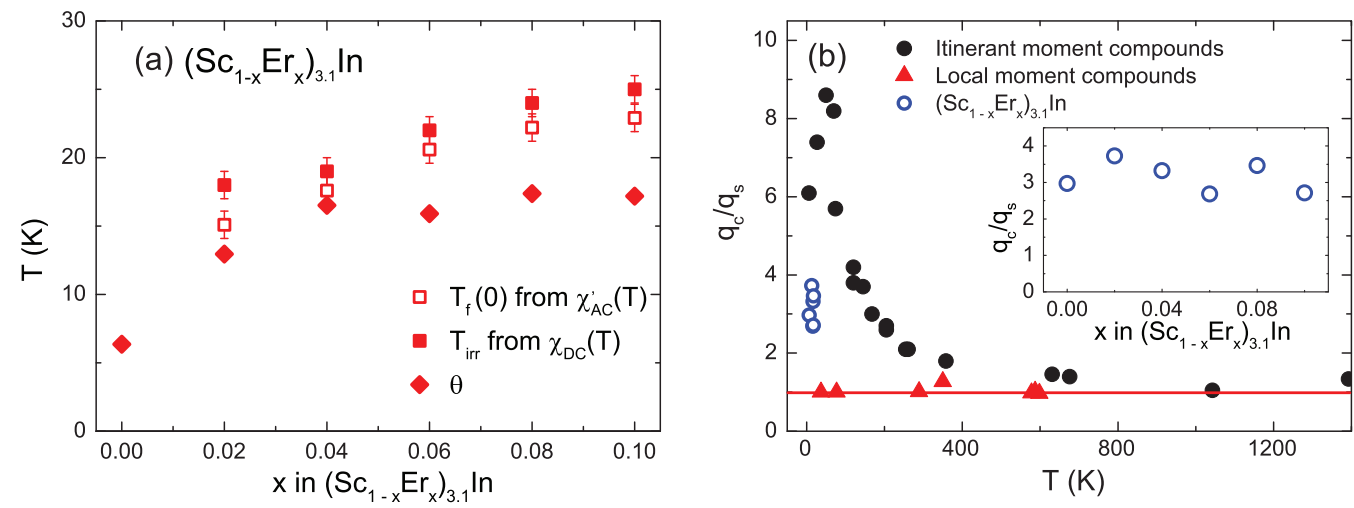

FIG. 7. (Color online) (a) The freezing temperature $T_{f}(0)$ (open squares), the irreversibility temperature $T_{\text {irr }}$ (full squares), and the Weiss-like temperature $\theta$ (full diamonds) as a function of $x$ in $\left(\mathrm{Sc}_{1-x} \mathrm{Er}_{x}\right)_{3.1} \mathrm{In}$. (b) RW plot for local moment compounds (triangles), itinerant moment compounds (full circles), and $\left(\mathrm{Sc}_{1-x} \mathrm{Er}_{x}\right)_{3.1} \mathrm{In}(0 \leqslant x \leqslant 0.10)$ (open circles). The red line indicates the local limit for $q_{c} / q_{s} \approx 1$. Inset: the RW ratio for $\left(\mathrm{Sc}_{1-x} \mathrm{Er}_{x}\right)_{3.1} \operatorname{In}(0 \leqslant x \leqslant 0.10)$.

$x>0, C_{p} / T$ versus $T^{2}$ (Fig. 6) displays a low- $T$ upturn, whose origin maybe be attributed to either a Schottky anomaly or NFL behavior. This remains to be clarified in a future study.

The composition dependence of the freezing temperature $T_{f}(0)$ and the irreversibility temperature $T_{\text {irr }}$ is shown as squares in Fig. 7. By contrast to the effects of $\mathrm{Gd}$ doping in $\mathrm{ZrZn}_{2},{ }^{7}$ where the Weiss-like temperature $\theta$ was suppressed with increasing amounts of local moment, Er doping of $\mathrm{Sc}_{3.1} \mathrm{In}$ resulted in an increase of $\theta$.

The paramagnetic moment $\mu_{\mathrm{PM}}^{\mathrm{exp}}$ and the saturated moment estimate $\mu_{5.6 \mathrm{~T}}$ can be used to determine the magnetic carrier per atom $q_{c}$ and $q_{s}{ }^{41}$ The $q_{c}$ parameter describes the behavior of the system for temperatures above the transition temperature, and is determined from the paramagnetic moment $\mu_{\mathrm{PM}}^{\text {expt }}$, using ${ }^{41}$

$$
q_{c}\left(q_{c}+1\right)=\frac{\left(\mu_{\mathrm{PM}}^{\mathrm{expt}}\right)^{2}}{4} .
$$

Similarly, $q_{s}$ is obtained from the saturation magnetization at temperatures below the transition temperature as $q_{s}=\mu_{5.6 \mathrm{~T}} / 2$, where $\mu_{5.6 \mathrm{~T}}$ is assumed to be close to the saturated moment given the small slope of the $M(H)$ isotherms in Fig. 3. If the number of carriers below the transition temperature $q_{s}$ is the same as the one above the transition temperature $q_{c}$, the Rhodes-Wohlfarth (RW) ratio is $q_{c} / q_{s} \sim 1$, indicating a local moment system. The other limiting case is that for $q_{c} / q_{s}>1$, observed in delocalized or itinerant magnets $\left[q_{c} / q_{s}=4\right.$ for $\mathrm{ZrZn}_{2}$ and 6.1 for $\mathrm{Sc}_{3}$ In (Ref. 41)]. The values of the RW ratio for local (full triangles) and itinerant (full circles) moment compounds, together with those for $\left(\mathrm{Sc}_{1-x} \mathrm{Er}_{x}\right)_{3.1}$ In (open circles), are shown in Fig. $7(\mathrm{~b})$. The coexistence of local and itinerant magnetic moments in Er-doped $\mathrm{Sc}_{3.1}$ In yields $\mathrm{RW}$ ratios between 2.7 and 3.7, as evidenced in the inset of Fig. 7(b). However, this ratio remains enhanced compared to the local moment limit for $x \leqslant 0.10$, suggesting that larger amounts of local moments would be necessary before the local moment magnetism became predominant.

\section{CONCLUSIONS}

A nonmagnetic dopant such as Lu (Ref. 14) yielded no glassy behavior in $\left(\mathrm{Sc}_{1-x} \mathrm{Lu}_{x}\right)_{3.1} \mathrm{In}$, but rather a gradual suppression of the ferromagnetic state towards a QCP. Conversely, Gd local moment doping of another itinerant ferromagnet with no magnetic moments $\mathrm{ZrZn}_{2}$ also resulted in a gradual suppression of the ferromagnetic state, with no evidence for the glassiness. ${ }^{7}$ Er doping in $\mathrm{Sc}_{3.1}$ In contrasts the findings for $\mathrm{Zr}_{1-x} \mathrm{Gd}_{x} \mathrm{Zn}_{2}$, as the paramagnetic moment is enhanced with increasing $x$ in the former, while the doping results in cluster glass behavior in $\left(\mathrm{Sc}_{1-x} \mathrm{Er}_{x}\right)_{3.1} \mathrm{In}$, with cooperative behavior of the local and itinerant moments suggested by the dc susceptibility $\chi$.

The ac and dc magnetization measurements, along with specific-heat data, show that $\left(\mathrm{Sc}_{1-x} \mathrm{Er}_{x}\right)_{3.1}$ In $(0.02 \leqslant x \leqslant$ $0.10)$ exhibits cluster-glass behavior below the characteristic freezing temperature $15 \mathrm{~K} \leqslant T_{f}(0) \leqslant 24 \mathrm{~K}$, which is enhanced by the increasing doping amount $x$. The clusterglass behavior is marked by the irreversibility of ZFC-FC dc magnetization data, a broad frequency-dependent peak in the ac susceptibility, a large value of $\delta$ parameter, the lack of saturation in the low-temperature magnetization at high fields, and a weak anomaly in the specific-heat data. Moreover, the Vogel-Fulcher analysis established that the clusters were strongly correlated.

Given that the emergence of cluster-glass state is only possible via frustration of the lattice, crystal-field-induced destabilization of magnetic moments, and site disorder, we propose site disorder and frustration to be at play here. The lattice of $\mathrm{Sc}_{3.1}$ In exhibits reduced dimensionality ${ }^{42}$ due to nearly one-dimensional bipyramidal Sc-In chains. The crystallographic frustration and site disorder, induced by $\mathrm{Er}$ doping on the bipyramidal sites, induces a cluster-glass state that originates from a metallic ferromagnetic ground state. It is imperious that the interplay between the local and itinerant moment in this system be elucidated, and neutron diffraction experiments are underway.

\section{ACKNOWLEDGMENT}

This work was supported by NSF Grant No. DMR 0847681. 
${ }^{1}$ M. Chefki, M. M. Abd-Elmeguid, H. Micklitz, C. Huhnt, W. Schlabitz, M. Reehuis, and W. Jeitschko, Phys. Rev. Lett. 80, 802 (1998).

${ }^{2}$ B. R. Coles, Physica B (Amsterdam) 91, 167 (1977).

${ }^{3}$ M. Shiga, H. Wada, K. Yoshimura, and Y. Nakamura, J. Magn. Magn. Mater. 54, 1073 (1986).

${ }^{4}$ M. D. Vannette, S. L. Bud'ko, P. C. Canfield, and R. Prozorov, J. Appl. Phys. 103, 07D302 (2008).

${ }^{5}$ B. T. Matthias and R. M. Bozorth, Phys. Rev. 109, 604 (1958).

${ }^{6}$ B. T. Matthias, A. M. Clogston, H. J. Williams, E. Corenzwit, and R. C. Sherwood, Phys. Rev. Lett. 7, 7 (1961).

${ }^{7}$ M. Asanuma and T. Yamadaya, J. Appl. Phys. 39, 1244 (1968).

${ }^{8}$ D. A. Sokolov, M. C. Aronson, W. Gannon, and Z. Fisk, Phys. Rev. Lett. 96, 116404 (2006).

${ }^{9}$ E. P. Wohlfarth and J. F. Cornwell, Phys. Rev. Lett. 7, 342 (1961).

${ }^{10}$ S. Curtarolo, D. Morgan, K. Persson, J. Rodgers, and G. Ceder, Phys. Rev. Lett. 91, 135503 (2003).

${ }^{11}$ J. Grewe, J. S. Schilling, K. Ikeda, and K. A. Gschneidner, Jr., Phys. Rev. B 40, 9017 (1989).

${ }^{12}$ W. E. Gardner, T. F. Smith, B. W. Howlett, C. W. Chu, and A. Sweedler, Phys. Rev. 166, 577 (1968).

${ }^{13}$ K. Ikeda and J. K. A. Gschneider, J. Magn. Magn. Mater. 22, 207 (1981).

${ }^{14}$ E. Svanidze and E. Morosan (unpublished).

${ }^{15}$ R. D. Shannon, Acta Crystallogr. 32, 751 (1976).

${ }^{16}$ J. A. Mydosh, Spin Glasses: An Experimental Introduction (Taylor and Francis, London, 1993).

${ }^{17}$ T. Nishioka, Y. Tabata, T. Taniguchi, and Y. Miyako, J. Phys. Soc. Jpn. 69, 1012 (2000).

${ }^{18}$ D. X. Li, T. Yamamura, S. Nimori, K. Yubuta, and Y. Shiokawa, Appl. Phys. Lett. 87, 142505 (2005).

${ }^{19}$ S. Süllow, G. J. Nieuwenhuys, A. A. Menovsky, J. A. Mydosh, S. A. M. Mentink, T. E. Mason, and W. J. L. Buyers, Phys. Rev. Lett. 78, 354 (1997).

${ }^{20}$ A. P. C. Mazumdar, Mater. Res. Bull. 46, 682 (2011).
${ }^{21}$ S. Riyadi, S. Giriyapura, R. A. de Groot, A. Caretta, P. H. M. van Loosdrecht, T. T. M. Palstra, and G. R. Blake, Chem. Mater. 23, 1578 (2011).

${ }^{22}$ S. Zhang, S. Tan, L. Pi, and Y. Zhang, J. Magn. Magn. Mater. 322, 3381 (2010).

${ }^{23}$ S. L. Huang, L. X. Guan, J. B. Yi, B. C. Zhao, Y. Wu, Z. C. Fan, T. C. Sum, J. Ding, and L. Wang, J. Appl. Phys. 104, 123904 (2008).

${ }^{24}$ T. Kato and T. Saita, Physica B (Amsterdam) 284, 1347 (2000).

${ }^{25}$ N. Marcano, J. C. GómezSal, J. I. Espeso, L. Fernández Barquín, and C. Paulsen, Phys. Rev. B 76, 224419 (2007).

${ }^{26}$ D. X. Li, S. Nimori, Y. Shiokawa, Y. Haga, E. Yamamoto, and Y. Onuki, Phys. Rev. B 68, 172405 (2003).

${ }^{27}$ M. Szlawska, D. Gnida, and D. Kaczorowski, Phys. Rev. B 84, 134410 (2011).

${ }^{28}$ V. K. Anand, D. T. Adroja, and A. D. Hillier, Phys. Rev. B 85, 014418 (2012).

${ }^{29}$ E. Svanidze, T. Besara, T. Siegrist, K. Han, and E. Morosan (unpublished).

${ }^{30}$ V. B. Compton and B. T. Matthias, Acta Crystallogr. 15, 94 (1962).

${ }^{31}$ T. Moriya, Spin Fluctuations in Itinerant Electron Magnetism (Springer, Berlin, 1985).

${ }^{32}$ K. Binder, Rev. Mod. Phys. 58, 801 (1986).

${ }^{33}$ J. Androulakis, N. Katsarakis, and J. Giapintzakis, J. Appl. Phys. 91, 9952 (2002).

${ }^{34}$ J. L. Dormann, L. Bessais, and D. Fiorani, J. Phys. C: Solid State Phys. 21, 2015 (1988).

${ }^{35}$ P. C. Hohenberg and B. I. Halperin, Rev. Mod. Phys. 49, 435 (1977).

${ }^{36}$ J. L. Tholence, Solid State Commun. 35, 113 (1980).

${ }^{37}$ G. S. Fulcher, J. Am. Ceram. Soc. 8, 339 (1925).

${ }^{38}$ J. Souletie and J. L. Tholence, Phys. Rev. B 32, 516 (1985).

${ }^{39}$ L. Yu, C. Qian, Q. Da-Wei, W. Ji-Liang, Z. Jing, W. Shuang, and G. Jing, Chin. Phys. B 20, 117502 (2011).

${ }^{40}$ S. Shtrikman and E. P. Wohlfarth, Phys. Lett. 85, 467 (1981).

${ }^{41}$ P. Rhodes and E. P. Wohlfarth, Proc. R. Soc. A 273, 247 (1963).

${ }^{42}$ T. Jeong, Solid State Commun. 138, 265 (2006). 